\title{
Innovative Astronomy Education Programs for Developing Countries
}

\author{
B.G. Sidharth, B.M. Birla Planetarium, Adarsh Nagar, \\ Hyderabad - 500463 India
}

\section{Introduction}

It is desirable that planetariums in developing countries should make the maximum and most efficient use of the planetarium infrastructure and facilities to cover as much ground as possible in the popularization and dissemination of astronomy. After all, the number of planetariums in developing countries necessarily has to be small, and so specialization in specific disciplines or fields becomes a luxury. In India, for example, there are about ten planetariums, and another five or six will come into operation in the next few years. But these planetariums have to cater to a large population. In the U.S.A., which has a fraction of India's population, on the other hand, there are hundreds of planetariums. The following suggestions are based on successfully implemented projects at the B.M. Birla Planetarium, Hyderabad.

A golden rule for planetarium programs anywhere, and certainly in developing countries, is to start a planetarium sky show or activity with a local flair. For example, the local names of stars and constellations, local myths, local astronomers or, more specifically, topics like the history of astronomy in the region should be highlighted.

\section{Planetarium Sky Shows}

It is important for planetariums in developing countries in particular to choose the levels of their astronomical presentations (sky shows) carefully. These could be broadly classified as follows:

a) Popular programs for the lay public. Such sky shows should have a minimum of subtle inputs. The lay audiences in developing countries definitely prefer a spectacle and a dramatic experience to a pedagogic presentation.

b) Sky shows, preferably live and participatory in nature, for school groups. It would be advisable to prepare them in consultation with the relevant teachers. In any case a dialogue with the teaching community is essential.

c) More specialized, again preferably live, programs for amateurs and astronomy students.

\section{Educational and Amateur Activities}

Educational activities should be spun around planetariums. It would be helpful if these planetariums also acquire mini/portable planetariums, and, of course, telescopes for such activities. At the B.M. Birla Planetarium, for example, a project entitled Astro School has caught the imagination of educators. In this program, a 
set of school children is given a one-day exposure to all the excitement of astronomy through sky shows, exposure to a mini planetarium, illustrated lectures, computer graphics, astronomical games, science-fiction films, and, whenever possible, sky observation sessions.

Planetariums in developing countries would also do well to organize informal astronomy courses for lay persons. At Hyderabad, the course is of a multi-media nature and lasts for three months, with two evening lectures per week. The course has generated so much enthusiasm that rejection of applicants is a major problem. The teaching community in schools, particularly in the rural areas, is in general scientifically impoverished and ill-equipped to teach astronomy or science itself while preserving the sense of excitement and discovery. So planetariums would render a service to the community by conducting two or three-day camps for science teachers from schools, particularly the rural schools. An important extension of this idea is camps of shorter duration, for example on selected Sundays, for the lay public. Such one-day camps using portable planetariums, for school students/teachers in the rural areas, would be a great boon for this less fortunate segment of society in the rural areas.

Regular lectures by interesting speakers, workshops - for example, on telescope making - and exhibition of astronomical and scientific films and videos should be a part of the culture of every planetarium. Such events are logically linked with the amateur astronomical activities that can build up around a planetarium.

It is interesting that at Hyderabad, where one of the only astronomy university departments in India has been functioning for nearly three decades, the first amateur astronomical association came into being shortly after the inauguration of the planetarium. The association was formed by a group of lay persons who attended a three-month multi-media astronomy course offered by the planetarium.

\section{Research Activities}

Seminars on catchy topics organized by the planetariums not only give a boost to the interest in astronomy but also earn for the institution a lot of publicity. This has been the case in Hyderabad, where the B.M. Birla Planetarium has organized major seminars on Halley's Comet, Indian Astronomy, and finally Ancient Astronomies. In fact, one of these seminars coincided with an equally important international seminar on a topic in theoretical physics. While the former got wide coverage in the press, the latter was almost totally ignored.

\section{Information Centers}

A planetarium would render its community a great service by organizing a research center for the history of astronomy of the region. Even the planetariumprojector facility itself could be directly used, for example, in the dating of historical events by using relevant astronomical allusions.

Planetariums in developing countries should act as centers for dissemination of astronomy information. At Hyderabad, this is done through posters, press releases 
and educational films which are telecast all over the country. In this connection, an acute problem faced by planetariums in developing countries is the lack of immediate access to important astronomical-event information. People look to planetariums, and not university departments, for information. And it is of vital importance that there should be an agency on the lines of the IAU telegrams that can transmit at a price, if necessary - such information, preferably in layman's language, to planetariums.

The planetarium at Hyderabad for example receives even long-distance calls from people wanting to know where and when a comet can be sighted and so on. So the importance of such an international information facility cannot be overemphasized.

Another sad problem faced by planetariums in developing countries is the lack of resources to meet the need for take-away literatures, audio tapes, video cassettes, planispheres, books, souvenirs, and so on. The problem is even more acute in a country like India with its import regulations.

\section{Leadership}

For developing countries, it is important that the established and successful planetariums should provide leadership for the new and also smaller planetariums. An acute problem in this context is the lack of trained personnel, for the simple reason that a planetarium culture is either non-existent or nearly so. With exactly this in mind, the B.M. Birla Planetarium, Hyderabad, has introduced a university-recognized post-graduate diploma in Planetarium Techniques and Management. This will, we hope, build up a cadre of planetarium directors and educators.

\section{Conclusion}

Lastly, it should be pointed out that the success of a planetarium depends to a great extent on its showmanship and marketing ability. In fact, this task is easier in the developing countries, where media coverage is much more accessible than in the advanced countries. An author once advised novices that they should break their necks to get into print. A good dictum for a planetarium would be that it should break its neck or whatever to remain in public view.

\section{Follow-up remarks by Joseph M. Chamberlain}

My colleagues on the panel have presented an excellent cross-section of activities and opportunities in the teaching of astronomy through the planetarium medium. Since our time allotment is brief, I do not want to duplicate, but I would like to summarize two very attractive programs that are offered in several planetar- 